\section{The cosmic molybdenum-neodymium isotope correlation and the building material of the Earth}

\section{J. Render ${ }^{1 *}$, M. Fischer-Gödde ${ }^{1}$, C. Burkhardt ${ }^{1}$, T. Kleine}

open 2 access

\section{Abstract}

The isotopic similarity of enstatite chondrites and Earth has often been cited as evidence that the Earth is made of enstatite chondrite-like material. Here we show, however, that enstatite chondrites exhibit nucleosynthetic molybdenum (Mo) isotope anomalies and, therefore, cannot represent the sole building blocks of the Earth. Enstatite and ordinary chondrites together with the Earth's mantle plot on a cosmic Mo-Nd isotope correlation line that reflects varying proportions of $s$-process matter in these samples. This correlation indicates that the nucleosynthetic makeup of Earth's building material did not change over time and that Earth, on average, accreted from bodies that originated closer to the Sun and were enriched in s-process matter compared to known chondrites. As such, any contribution of chondrites to Earth's accreting material must be compensated by the addition of $s$-process enriched bodies. This material is not present in our meteorite collections, but may have been sampled by Venus or Mercury. The $s$-process enriched nature of the Earth can fully account for by Venus or tion of Earth's mantle.

Received 9 September 2016 | Accepted 20 March 2017 | Published 14 April 2017

\section{Introduction}

Earth probably formed through the collision of numerous planetesimals and planetary embryos. Remnants of these bodies may today still reside in the asteroid belt and so some of Earth's building material might have been sampled by meteorites. This raises the question of whether meteorites are representative for the entire suite of bodies accreted by the Earth over time. Addressing this issue is critical not only for understanding the accretion history of the Earth, but also for constraining its chemical composition and early differentiation.

\footnotetext{
1. Institut für Planetologie, University of Münster, Wilhelm-Klemm-Str. 10, 48149 Münster, Germany
} Corresponding author (email: jan.render@uni-muenster.de)
The most powerful constraints for establishing potential genetic links between meteorites and the Earth come from nucleosynthetic isotope anomalies, which arise through the heterogeneous distribution of presolar matter in the solar protoplanetary disk. These isotopic variations have been documented for several elements, including lithophile (e.g., Cr, Ti, Nd; Carlson et al., 2007; Trinquier et al., 2007, 2009; Burkhardt et al., 2016) as well as moderately siderophile (e.g., Ni, Mo; Dauphas et al., 2002; Regelous et al., 2008; Burkhardt et al., 2011) and highly siderophile elements (e.g., Ru; Chen et al., 2010; Fischer-Gödde et al., 2015). As the present-day mantle composition of these elements has been set during different stages of Earth's accretion, the nucleosynthetic isotope signatures do not only provide information on whether or not specific meteorites are suitable building blocks of the Earth, but they also make it possible to identify potential changes in the nature of Earth's building material over time (Dauphas et al. 2004; Dauphas, 2017)

Several prior studies attempted to constrain the origin of Earth's building material by assessing which combination of known meteorites yields the isotopic composition of Earth's mantle (e.g., Dauphas et al., 2014; Dauphas, 2017). As enstatite chondrites are the only meteorite class for which no or only very small nucleosynthetic isotope anomalies are observed (Trinquier et al., 2007, 2009; Regelous et al., 2008; Dauphas et al., 2014), such models typically predict that Earth largely consists of enstatite chondrite-like material. This enstatite chondriteEarth model was initially proposed based on the similar O isotopic compositions of Earth and enstatite chondrites (Javoy, 1995), but gained additional strength owing to the isotopic similarity between these two bodies observed for several other elements (Javoy et al., 2010). Because enstatite chondrites and Earth's upper mantle are chemically quite different, however, the enstatite chondrite-Earth model in its original form requires that the Earth's mantle is chemically stratified (Javoy et al., 2010). To avoid these complications, more recent versions of this model have argued that enstatite chondrites do not represent the actual building blocks of the Earth, but simply sample the same isotopic solar nebula reservoir as the bodies accreted by the Earth (Dauphas et al., 2014; Dauphas, 2017).

All these prior models are based on the predication that meteorites are representative of the entire material that was available to build the larger embryos and terrestrial planets. This assumption is questionable, however, given that most meteorites probably formed at greater heliocentric distances than the main building material of the Earth. As such, it has also been argued that the building material of the Earth is not represented by known meteorites, but that it has been entirely incorporated into the terrestrial planets (Drake and Righter, 2002; Burkhardt et al., 2011, 2016). From an isotopic standpoint this model is difficult to test, because for most elements (e.g., $\mathrm{O}, \mathrm{Cr}$, Ti) the isotopic composition of the Earth is intermediate between the isotopic variations measured for meteorites. However, for Mo this does not seem to be the case and almost all meteorites are characterised by a deficit of Mo produced in the slow neutron capture process (s-process) of stellar nucleosynthesis (Dauphas et al., 2002; Burkhardt et al., 2011). Until now, enstatite chondrites and IAB iron meteorites are the only meteorites for 
which no clearly resolved Mo isotope anomalies have been identified, although there are hints of a small deficit in s-process Mo in enstatite chondrites as well (Burkhardt et al., 2011). The presence of such a Mo s-process deficit in enstatite chondrites, if confirmed, would place strict constraints on the possible contribution of enstatite chondrite-like material accreted by the Earth, and would allow assessment of the more general question of whether meteorites are representative of the building material of the Earth. To address these issues, we obtained new high-precision Mo isotopic data for enstatite chondrites.

\section{Mo Isotope Anomalies in Enstatite Chondrites \\ and the Cosmic Mo-Nd Isotope Correlation}

Eight enstatite chondrites as well as three ordinary and four CV chondrites were selected for this study. Details about sample preparation, the purification of Mo from the sample matrix, and the Mo isotope measurements using the ThermoScientific Neptune Plus MC-ICP-MS at Münster are given in the Supplementary Information. The Mo isotopic compositions of the chondrites were normalised to the terrestrial ${ }^{98} \mathrm{Mo} /{ }^{96} \mathrm{Mo}$ using the exponential law, and are reported in $\mu^{\mathrm{i}} \mathrm{Mo}$ values as the parts per million deviation from the compositions measured for the Alfa Aesar Mo standard solution. Each sample was analysed multiple times and the $\mu^{\mathrm{i}} \mathrm{Mo}$ values reported in Table 1 represent mean values for pooled solution replicates $(\mathrm{N}=3-5)$ together with their associated uncertainties. The accuracy and precision of the measurements were assessed by replicate digestions and multiple measurements of the USGS Hawaiian basalt BHVO-2, for which we obtained $\mu^{\mathrm{i}} \mathrm{Mo} \sim 0$ (Table 1 ).

The new data for enstatite and ordinary chondrites show well-resolved Mo isotope anomalies relative to the terrestrial solution standard (i.e. $\mu^{i} \mathrm{Mo}=0$ ) and the rock standard $\mathrm{BHVO}-2$ that was processed and analysed together with the chondrite samples (Table 1, Fig. 1). The anomalies are most pronounced for $\mu^{92} \mathrm{Mo}$ and $\mu^{94} \mathrm{Mo}$, for which most (6 out of 8) enstatite chondrites show resolved excesses (Fig. 1). The mean composition of all eight investigated enstatite chondrites display well-resolved positive $\mu^{\mathrm{i} M o}$ anomalies for all Mo isotopes (Fig. 1). Compared to the enstatite chondrites, the ordinary chondrites exhibit larger Mo isotope anomalies, consistent with results of a prior study (Burkhardt et al., 2011). As is evident from plots of $\mu^{i}$ Mo versus $\mu^{92} \mathrm{Mo}$, the Mo isotope anomalies are correlated as expected for the heterogeneous distribution of s-process matter indicating that enstatite and ordinary chondrites are characterised by a deficit in s-process Mo compared to Earth's mantle (Fig. S-3).

As for Mo, the $\mathrm{Nd}$ isotope compositions of chondrites also reveal an increasing deficit in $s$-process isotopes from enstatite to ordinary chondrites (Burkhardt et al., 2016). The Mo and Nd isotope anomalies in these chondrites plot on a single correlation line together with the composition of Earth's mantle (Fig. 2). Of note, the Mo-Nd isotope correlation holds regardless of which $\mathrm{Nd}$ isotope is used (Figs. 2, S-4). As both the Mo and Nd isotope variations reflect a deficit in s-process matter, this cosmic Mo-Nd isotope correlation most likely results from the heterogeneous distribution of a single presolar carrier enriched in s-process $\mathrm{Mo}$ and $\mathrm{Nd}$.

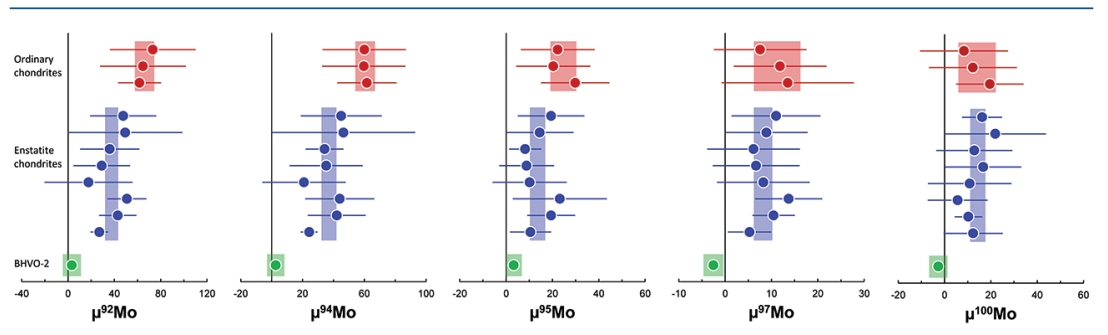

Figure 1 Mo isotopic composition of enstatite and ordinary chondrites. Error bars indicate external uncertainties derived from the 2 s.d. obtained for terrestrial rock standards analysed in this study (if $\mathrm{N}<4$ ) or Student's t-values $95 \%$ confidence interval of multiple solution replicates of a sample (if $\mathrm{N} \geq 4$ ) (Table 1). Shaded areas indicate mean compositions of enstatite and ordinary chondrites as well as the terrestrial rock standard BHVO-2 with their Student's t-values $95 \%$ confidence intervals.
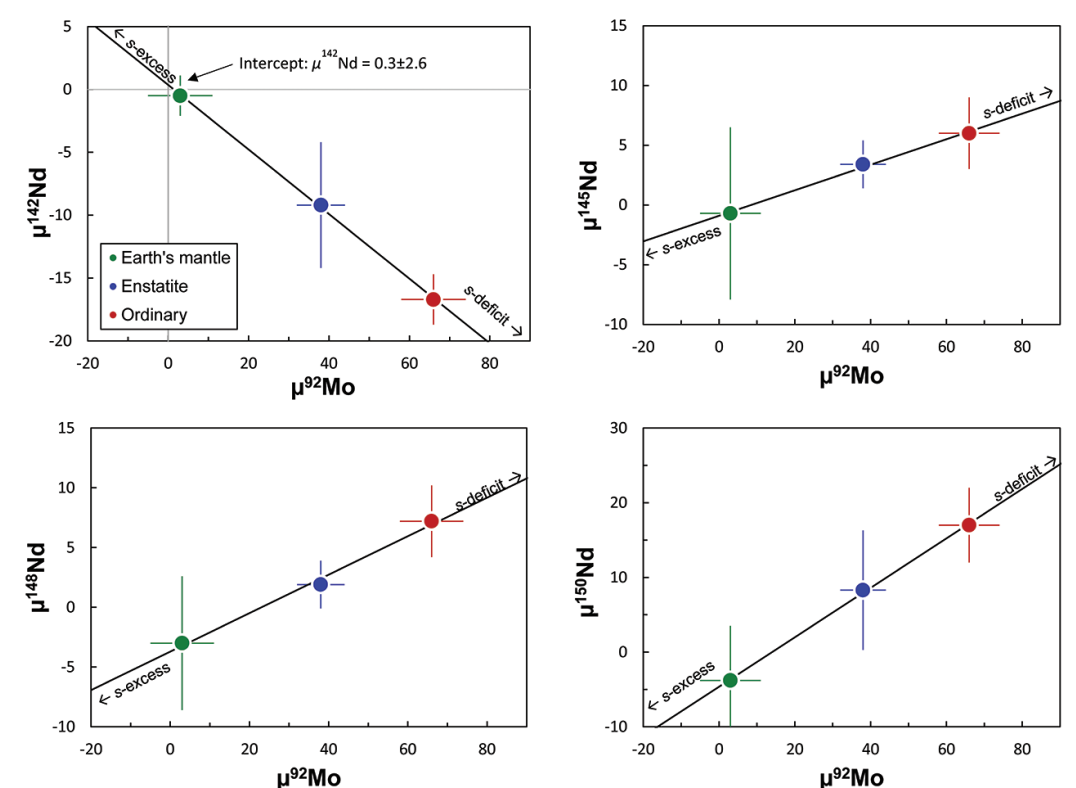

Figure 2 Cosmic Mo-Nd isotope correlation. Mo isotopic data for enstatite and ordinary chondrites from this study, Nd isotopic data are from Burkhardt et al. (2016). For a plot with carbonaceous chondrites included see Figure S-4. Regression of $\mu^{142} \mathrm{Nd}$ versus $\mu^{92} \mathrm{Mo}$ calculated using IsoPlot. The good alignment of the data combined with the observation that both ${ }^{142} \mathrm{Nd}$ between Earth's mantle and chondrites is solely nucleosynthetic in origin. 
Table 1 Molybdenum isotopic compositions of enstatite and ordinary chondrites.

\begin{tabular}{|c|c|c|c|c|c|c|c|}
\hline Sample & Type & $\mathrm{N}^{\mathrm{a}}$ & $\mu^{92} \mathbf{M o}^{\mathrm{b}}$ & $\mu^{94} \mathbf{M o}^{\mathrm{b}}$ & $\mu^{95} \mathbf{M o}^{\mathrm{b}}$ & $\mu^{97} \mathbf{M o}^{\mathrm{b}}$ & $\mu^{100} \mathbf{M o}^{\mathrm{b}}$ \\
\hline BHVO-2 & Basalt & $24(3)$ & $3 \pm 8$ & $3 \pm 6$ & $3 \pm 3$ & $-3 \pm 2$ & $-3 \pm 4$ \\
\hline \pm 2 s.d. & & & \pm 37 & \pm 27 & \pm 16 & \pm 10 & \pm 19 \\
\hline \multicolumn{8}{|l|}{ Enstatite chondrites } \\
\hline $\begin{array}{l}\text { LON } 94100 \\
\text { LAP } 10130 \\
\text { MAC } 02747 \\
\text { TIL } 91714 \\
\text { ALHA } 81021 \\
\text { Khairpur } \\
\quad \text { EL mean } \\
\text { EET } 87747 \\
\text { Abee } \\
\quad \text { EH mean } \\
\quad \text { Mean }(\text { EH, EL) }\end{array}$ & $\begin{array}{l}\text { EL6 } \\
\text { EL6 } \\
\text { EL4 } \\
\text { EL5 } \\
\text { EL6 } \\
\text { EL6 } \\
\text { EH4 } \\
\text { EH4 }\end{array}$ & $\begin{array}{c}3 \\
5(2) \\
5 \\
4 \\
5 \\
7(2) \\
29 \\
5 \\
5 \\
10 \\
39\end{array}$ & $\begin{array}{c}18 \pm 37 \\
51 \pm 17 \\
36 \pm 26 \\
29 \pm 24 \\
43 \pm 16 \\
27 \pm 8 \\
35 \pm 6 \\
48 \pm 29 \\
49 \pm 8 \\
49 \pm 11 \\
38 \pm 6\end{array}$ & $\begin{array}{l}21 \pm 27 \\
44 \pm 22 \\
34 \pm 12 \\
35 \pm 24 \\
42 \pm 19 \\
24 \pm 5 \\
33 \pm 5 \\
45 \pm 26 \\
46 \pm 10 \\
46 \pm 11 \\
36 \pm 5\end{array}$ & $\begin{array}{c}10 \pm 16 \\
23 \pm 20 \\
8 \pm 7 \\
9 \pm 12 \\
19 \pm 10 \\
11 \pm 9 \\
13 \pm 4 \\
19 \pm 14 \\
15 \pm 9 \\
17 \pm 7 \\
14 \pm 3\end{array}$ & $\begin{array}{c}8 \pm 10 \\
14 \pm 7 \\
6 \pm 10 \\
7 \pm 9 \\
10 \pm 5 \\
5 \pm 5 \\
8 \pm 2 \\
11 \pm 10 \\
9 \pm 9 \\
10 \pm 5 \\
9 \pm 2\end{array}$ & $\begin{array}{c}11 \pm 19 \\
6 \pm 13 \\
13 \pm 16 \\
17 \pm 16 \\
10 \pm 6 \\
12 \pm 13 \\
12 \pm 4 \\
16 \pm 9 \\
22 \pm 12 \\
19 \pm 6 \\
14 \pm 3\end{array}$ \\
\hline \multicolumn{8}{|l|}{ Ordinary chondrites } \\
\hline $\begin{array}{l}\text { Braunschweig } \\
\text { Kunashak } \\
\text { Gao Guenie } \\
\quad \text { Mean }(\mathrm{L}, \mathrm{H})\end{array}$ & $\begin{array}{l}\text { L5 } \\
\text { L5 } \\
\text { H5 }\end{array}$ & $\begin{array}{c}3 \\
3 \\
4 \\
10\end{array}$ & $\begin{array}{c}73 \pm 37 \\
65 \pm 37 \\
62 \pm 19 \\
66 \pm 8\end{array}$ & $\begin{array}{c}60 \pm 27 \\
60 \pm 27 \\
62 \pm 19 \\
61 \pm 6\end{array}$ & $\begin{array}{c}22 \pm 16 \\
20 \pm 16 \\
30 \pm 15 \\
25 \pm 6\end{array}$ & $\begin{array}{c}8 \pm 10 \\
12 \pm 10 \\
13 \pm 14 \\
11 \pm 5\end{array}$ & $\begin{array}{c}8 \pm 19 \\
12 \pm 19 \\
20 \pm 15 \\
14 \pm 8\end{array}$ \\
\hline
\end{tabular}

${ }^{a}$ Number of analyses of the same sample; number in parenthesis indicates number of replicate digestions. ${ }^{b}$ Molybdenum isotope data are mass-bias corrected by normalising to ${ }^{98} \mathrm{Mo} /{ }^{16} \mathrm{Mo}=1.453171$ and using the $\left.\mu^{i} \mathrm{Mo} /{ }^{6} \mathrm{Mo}_{\text {standard }}-1\right) \times 10^{6}$. Uncertainties for samples measured more than three times are given as Student's $t$-values $95 \%$ confidence intervals from replicate analyses of the same sample. For samples measured $n \leq 3$ times (LON 94100 , Braunschweig. Kunashak), quoted uncertainties are given as external uncertainties as defined by the standard deviation ( 2 s.d.) of repeated analyses from replicate digestions of BHVO-2 reference samples.

In spite of their large Mo isotope anomalies, bulk CV chondrites do not show significant nucleosynthetic $\mathrm{Nd}$ isotope anomalies and therefore do not plot on the Mo-Nd isotope correlation (Fig. S-4). Burkhardt et al. (2016) attributed the absence of clearly resolvable nucleosynthetic Nd isotope anomalies in CV chondrites to the admixture of isotopically anomalous $\mathrm{Ca}$-Al-rich inclusions (CAIs) to these samples. Mathematical removal of CAIs from the measured bulk CV composition shows that a hypothetical CAI-free CV chondrite would not only have a large s-process deficit for $\mathrm{Nd}\left(\right.$ e.g., $\mu^{150} \mathrm{Nd} \approx 50$ ), but would also plot on the Mo-Nd isotope correlation together with enstatite and ordinary chondrites (Fig. S-4). Thus, the displacement of CV chondrites from the Mo-Nd isotope correlation most likely reflects the admixture of CAIs to these samples.

\section{Nature of Earth's Building Material}

There is no a priori reason why the Earth's mantle would plot on a single Mo-Nd correlation line together with enstatite and ordinary chondrites. This is because, as a moderately siderophile element, most of the Mo present in Earth's mantle derives from the last 10-20\% of accretion (Walker et al., 2015; Dauphas, 2017) whereas $\mathrm{Nd}$, as a lithophile element, records the average composition of the material added to the Earth during its entire growth history. Thus, Mo and Nd record different stages of Earth's accretion and any change in the isotopic composition of the accreting material would have affected the Mo and Nd isotopic compositions of Earth's mantle differently. For instance, the Mo isotopic data would be consistent with accretion of the first $~ 80-90 \%$ of the Earth from enstatite chondrite-like material (which would have left no impact on the Mo isotopic composition of the present-day Earth's mantle because almost all of that Mo would have been sequestrated into the core), followed by the accretion of the final $\sim 10-20 \%$ of distinct material characterised by $\mu^{92} \mathrm{Mo}=0$. In this scenario, the $\mathrm{Nd}$ isotopic composition of Earth's mantle would predominantly record the first $\sim 80-90 \%$ of accretion and would, therefore, be enstatite chondrite-like. However, in this case the Earth's mantle would not plot on a Mo-Nd isotope correlation line together with enstatite and ordinary chondrites. Instead, the observed correlation of Mo and Nd isotopes (Fig. 2) indicates that the nucleosynthetic signatures and hence, genetic makeup of Earth's building material did not change significantly as accretion proceeded.

Akin to the Mo-Nd correlation observed here, meteorites define a cosmic $\mathrm{Mo}-\mathrm{Ru}$ isotope correlation that also reflects variable s-process deficits in the meteorites (Dauphas et al., 2004). As a highly siderophile element, Ru predominantly reflects the addition of primitive material to the Earth's mantle after the cessation of core formation (the 'late veneer'; e g. Walker et al. 2015). Of note enstatite chondrites also show Ru isotope anomalies (e.g., $\mu^{100} \mathrm{Ru}=-8 \pm 4$ ), which, as for Mo and Nd, reflect a deficit in s-process matter compared to Earth's mantle (Fischer-Gödde and Kleine, 2017).

Taken together, the Mo, Nd and Ru isotopic data demonstrate that throughout its entire accretion history the building material of the Earth, including the late veneer, was on average characterised by an excess in s-process matter compared to chondrites, including enstatite chondrites. As such, the enstatite chondrite-Earth model loses its viability. This model in its original form (Javoy, 1995; Javoy et al., 2010) was based on the observation that enstatite chondrites are the only meteorites not showing mass-independent isotope anomalies compared to Earth. However, with the new Mo, $\mathrm{Nd}$ and Ru isotopic data, this basic assumption no longer holds true.

Dauphas (2017), using nucleosynthetic isotope anomalies for several other elements, also argued that the genetic heritage of Earth's accreting material did not change over time. By assuming that Earth's building material consisted of four components whose isotopic compositions are represented by enstatite, ordinary 
as well as CI and CV/CO chondrites, Dauphas (2017) calculated that the isotopic composition of Earth's mantle is best reproduced if the Earth accreted almost exclusively from enstatite chondrite-like material throughout its accretion history However, with the new Mo isotopic data of the present study, and the cosmic Mo-Nd isotope correlation, it becomes clear that no combination of known chondrites results in the Mo and Nd isotopic compositions of Earth's mantle. This is because all chondrites, including enstatite chondrites, are characterised by a deficit in s-process nuclides compared to Earth's mantle (Fig. 2). Consequently, the Earth cannot solely consist of known meteorites but instead accreted from material that on average was characterised by an excess in s-process matter compared to known meteorites. This does not exclude that meteorites contributed to the building material of the Earth, but any such contribution must be balanced by the accretion of $s$-process enriched material to the Earth. This material is not present in our current meteorite collections, but, if it existed, might have been sampled by Mercury and Venus (Fig. 3). We note, however, that the isotopic data taken together neither require nor exclude the contribution of meteorites to the building material of the Earth.

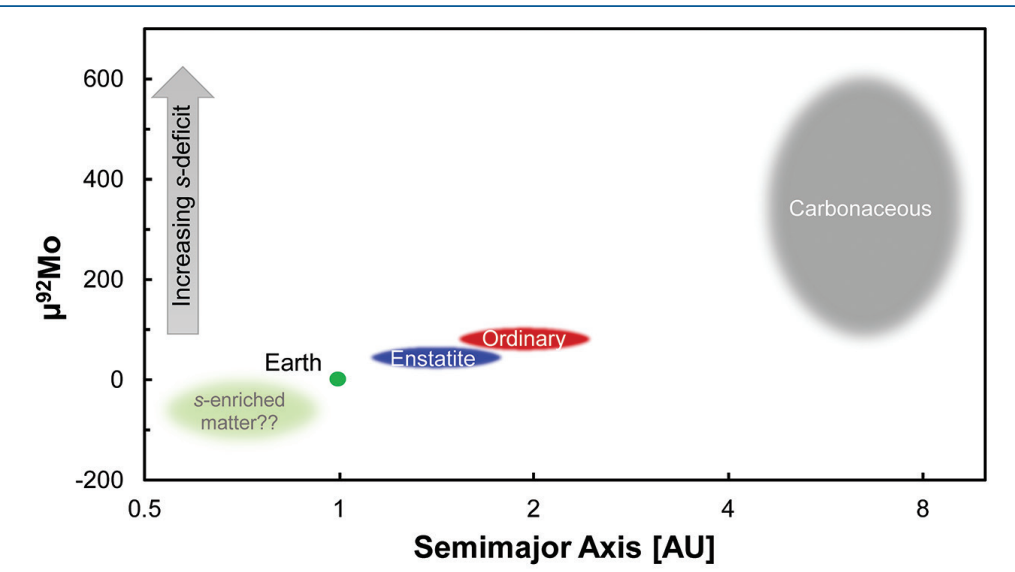

Figure $3 \mu^{92} \mathrm{Mo}$ versus heliocentric distance. Range of $\mu^{92} \mathrm{Mo}$ for carbonaceous chondrites from Burkhardt et al. (2011). Heliocentric distances are based on the Grand Tack model, in which bodies between 3 and 6 AU were largely incorporated into Jupiter.

The s-process enriched nature of the Earth compared to chondrites has important implications for ${ }^{146} \mathrm{Sm} \_{ }^{142} \mathrm{Nd}$ chronometry. Different $\mu^{142} \mathrm{Nd}$ compositions of chondrites and the Earth's mantle were initially interpreted to result from a very early global differentiation of the silicate Earth (e.g., Boyet and Carlson, 2005). However, on the basis of correlations for non-radiogenic Nd isotope anomalies, Burkhardt et al. (2016) recently showed that this ${ }^{142} \mathrm{Nd}$ difference is nucleosynthetic in origin. The observation that the Mo-Nd isotope correlation lines defined by Earth's mantle together with enstatite and ordinary chondrites include the $\mu^{142} \mathrm{Nd}-\mu^{92} \mathrm{Mo}$ correlation confirms this finding. The intercept of this correlation at $\mu^{92} \mathrm{Mo}=0$ is $\mu^{142} \mathrm{Nd}=0.3 \pm 2.6$, demonstrating that once nucleosynthetic isotope variations are taken into account, the Earth's mantle has no radiogenic ${ }^{142} \mathrm{Nd}$ anomaly relative to chondrites and that, therefore, no early differentiation is required to account for the present-day ${ }^{142} \mathrm{Nd}$ composition of the accessible Earth's mantle.

\section{Acknowledgements}

We thank the Meteorite Working Group (NASA), the National History Musuem (London) and R. Bartoschewitz for providing samples. Constructive reviews from two anonymous referees and editor G. Pearson helped improve the manuscript and are gratefully acknowledged. We also thank G. Budde for assistance with lab work and MC-ICP-MS measurements. This work was funded by the Deutsche Forschungsgemeinschaft (SFB-TRR 170, subproject B3-1). J.R. was partially supported by the Deutsche Forschungsgemeinschaft (grant KL 1857/4 to T.K.) and C.B. was supported by the European Research Council Consolidator Grant ISOCORE to T.K. (grant agreement no. 616564). This is TRR 170 publication no. 22.

Editor: Graham Pearson

\section{Additional Information}

Supplementary Information accompanies this letter at www.geochemicalperspectivesletters.org/article1720

Reprints and permission information are available online at http://www. geochemicalperspectivesletters.org/copyright-and-permissions

Cite this letter as: Render, J., Fischer-Gödde, M., Burkhardt, C., Kleine, T. (2017) The cosmic molybdenum-neodymium isotope correlation and the building material of the Earth. Geochem. Persp. Let. 3, 170-178.

\section{References}

BOYET, M., CARLSON, R.W. (2005) 142Nd evidence for early ( $>4.53 \mathrm{Ga}$ ) global differentiation of the silicate Earth. Science 309, 576-581.

Burkhardt, C., Kleine, T., Oberli, F., Pack, A., Bourdon, B., Wieler, R. (2011) Molybdenum isotope anomalies in meteorites: Constraints on solar nebula evolution and origin of the Earth. Earth and Planetary Science Letters 312, 390-400.

Burkhardt, C., Borg, L.E., Brennecka, G.A., Shollenberger, Q.R., Dauphas, N., Kleine, T. (2016) A nucleosynthetic origin for the Earth's anomalous ${ }^{142} \mathrm{Nd}$ composition. Nature 537, 394-398. 
CARLSON, R.W., BOYET, M., Horan, M.F. (2007) Chondrite barium, neodymium, and samarium isotopic heterogeneity and early earth differentation. Science 316, 1175-1178.

Chen, J.H., Papanastassiou, D.A., WasserburG, G.J. (2010) Ruthenium endemic isotope effects in chondrites and differentiated meteorites. Geochimica et Cosmochimica Acta 74, 3851-3862.

DAUPHAS, N. (2017) The isotopic nature of the Earth's accreting material through time. Nature 541, $521-524$.

DAUPHAS, N., MARTY, B., ReISBERG, L. (2002) Molybdenum nucleosynthetic dichotomy revealed in primitive meteorites. Astrophysical Journal 569, L139-L142.

Dauphas, N., DAVIS, A.M., MARTY, B., ReisberG, L. (2004) The cosmic molybdenum-ruthenium isotope correlation. Earth and Planetary Science Letters 226, 465-475.

Dauphas, N., Chen, J.H., Zhang, J., Papanastassiou, D.A., Davis, A.M., Travaglio, C. (2014) Calcium-48 isotopic anomalies in bulk chondrites and achondrites: Evidence for a uniform isotopic reservoir in the inner protoplanetary disk. Earth and Planetary Science Letters 407, 96-108.

Drake, M.J., Righter, K. (2002) Determining the composition of the Earth. Nature 416, 39-44.

FisCHER-GÖDDE, M., BURKHARDT, C., KRUIJER, T.S., KLEINE, T. (2015) Ru isotope heterogeneity in the solar protoplanetary disk. Geochimica et Cosmochimica Acta 168, 151-171.

FisCHER-GöDDE, M., KLEINE, T. (2017) Ruthenium isotopic evidence for an inner Solar System origin of the late veneer. Nature 541, 525-527.

JAVOY, M. (1995) The integral enstatite chondrite model of the Earth. Geophysical Research Letters 22 2219-2222.

Javoy, M., Kaminski, E., Guyot, F., Andrault, D., Sanloup, C., Moreira, M., Labrosse, S. Jambon, A., Agrinier, P., Davaille, A., JaUpart, C. (2010) The chemical composition of the Earth: Enstatite chondrite models. Earth and Planetary Science Letters 293, 259-268.

Regelous, M., Elliott, T., CoATH, C.D. (2008) Nickel isotope heterogeneity in the early Solar System. Earth and Planetary Science Letters 272, 330-338.

Trinquier, A., BiRCK, J.L., ALLĖGRE, C.J. (2007) Widespread Cr-54 heterogeneity in the inner solar system. Astrophysical Journal 655, 1179-1185.

Tringuier, A., Elliott, T., Ulfbeck, D., Coath, C., Krot, A.N., Bizzarro, M. (2009) Origin of Nucleosynthetic Isotope Heterogeneity in the Solar Protoplanetary Disk. Science 324, 374-376.

Walker, R.J., Bermingham, K., Liu, J., Puchtel, I.S., Touboul, M., Worsham, E.A. (2015) In search of late-stage planetary building blocks. Chemical Geology 411, 125-142. 